\title{
ORTHOGONAL GROUP MATRICES OF HYPEROCTAHEDRAL GROUPS
}

\author{
J. S. FRAME
}

To the memory of TADASI NaKayama

1. Introduction. The hyperoctahedral group $G_{n}$ of order $2^{n} n$ ! is generated by permutations and sign changes applied to $n$ digits, $d=1,2, \ldots, n$. The $2^{n}$ sign changes generate a normal subgroup $\Sigma_{n}$ whose factor group $G_{n} / \Sigma_{n}$ is isomorphic with the symmetric group $S_{n}$ of order $n$ !. To each irreducible orthogonal representation $\{\lambda ; \mu\}$ of $G_{n}$ corresponds an ordered pair of partitions [ $\lambda]$ of $l$ and [ $\mu$ ] of $m$, where $l+m=n$. The faithful representation $\{n-1 ; 1\}$ of $G_{n}$ is the real monomial group $R_{n}$ of degree $n$. The representations $\{\lambda ; 0\}$ of $G_{n}$ with $l=n, m=0$, are isomorphic with corresponding irreducible representations $\{\lambda\}$ of $S_{n}$. If the representation $\{\lambda ; \mu\}$ maps the element $g_{k}$ of $G_{n}$ into the real orthogonal matrix $M^{\lambda \mu}\left(g_{k}\right)$ of degree $f^{\lambda \mu}$, we define the group matrix of $\{\lambda ; \mu\}$ to be

$$
\mathfrak{M}^{\lambda \mu}=\sum_{k} g_{k}^{-1} M^{\lambda \mu}\left(g_{k}\right) \quad g_{k} \in G_{n}
$$

Our purpose is to determine explicitly for each $\{\lambda ; \mu\}$ the $u v$-entry of the group matrix of an irreducible orthogonal representation of $G_{n}$, and incidentally those of $S_{n}$, in the form

$$
\mathfrak{M}_{u v v}^{\lambda u}=\gamma_{v} E^{\lambda \mu} \sigma^{\lambda \mu} \gamma_{u}^{-1}
$$

by describing in the group ring $\Gamma$ of $G_{n}$ a suitab'e pair of ring elements $E^{\lambda \mu}$ related to permutations of $S_{n}$, and $\sigma^{\lambda \mu}$ related to sign changes of $\Sigma_{n}$, and also a set of invertible ring factors $\gamma_{v}$ that meet our requirements. Matrices $M^{\lambda 0}\left(\tau_{d}\right)$ for transpositions $\tau_{d}$ of consecutive digits $d, d+1$ are to be those of Young's orthogonal representation $\{\lambda\}$ of $S_{n}[4]$. The matrix $M^{\lambda \mu}\left(\sigma_{d}\right)$ for the element $\sigma_{d}$ of $\Sigma_{n}$ that changes the sign of the digit $d$ is to be a diagonal matrix with $v v$-entry +1 or -1 according as the digit $d$ is assigned to the

Received July, 1965. 
first part $[\lambda]$ or the second part $[\mu]$ in the $v^{t h}$ standard tableau $t_{v}^{\lambda \mu}$ for the diagram $[\lambda ; \mu]$.

2. Properties of the group matrix. For any group matrix $\mathfrak{M}$ we have the relations [2]

$$
\begin{aligned}
g \mathfrak{M} & =\sum_{k} g g_{k}^{-1} M\left(g_{k}\right)=\sum_{k} g\left(g_{k} g\right)^{-1} M\left(g_{k} g\right)=\mathfrak{M} M(g) \\
\mathfrak{M}^{T} \mathfrak{M}^{T} & =\sum_{h} \sum_{k} g_{h}^{-1} M^{T^{T}}\left(g_{h}\right) g_{k}^{-1} M^{T}\left(g_{k}\right)=\sum_{h, k}\left(g_{k} g_{h}\right)^{-1} M^{T}\left(g_{k} g_{i}\right)={ }^{o} G \mathbb{M}^{T}
\end{aligned}
$$

where ${ }^{o} G$ denotes the order of $G$. Since any element $g_{k}$ of $G_{n}$ can be factored into transpositions $\tau_{d}$ of consecutive digits $d, d+1$, and sign changes $\sigma_{d}$, we require the following conditions on $E^{\lambda \mu} \sigma^{\lambda \mu}=E \sigma$ and $\gamma_{v}$ in (1.2). To simplify the formulas we omit the superscripts $\lambda, \mu$.

$$
\begin{aligned}
& \tau_{d} \gamma_{v} E \sigma \gamma_{t}^{-1}=\sum_{u} \gamma_{u} E \sigma \gamma_{t}^{-1} M_{u v}\left(\tau_{d}\right) \\
& \sigma_{d} \gamma_{v} E_{\sigma \gamma_{t}^{-1}}=\gamma_{v} E_{\sigma \gamma_{t}^{-1}} M_{v v}\left(\sigma_{d}\right) \\
& \sum_{t=1}^{f} \gamma_{u} E \sigma \gamma_{t}^{-1} \gamma_{t} E_{\sigma r_{v}}{ }^{-1}={ }^{o} G\left(\gamma_{u} E \sigma \gamma_{v}^{-1}\right)
\end{aligned}
$$

Conditions (2.3), (2.4), and (2.5) are satisfied if

$$
\begin{aligned}
\tau_{d} \gamma_{v} & =\sum_{u} \gamma_{u} M_{u v}\left(\tau_{d}\right) \\
\sigma_{d} \gamma v & =\gamma_{v} M_{v v}\left(\sigma_{d}\right) \\
E^{2} & =\left({ }^{o} G / f\right) E=h E \\
\sigma E & =E \sigma, \sigma^{2}=\sigma .
\end{aligned}
$$

The requirement that the representation $M$ be orthogonal is satisfied if the coefficient of $g_{k}^{-1}$ in $\gamma_{v} E_{\sigma} \gamma_{u}^{-1}$ is the same as that of $g_{k}$ in $\gamma_{u} \sigma E{ }_{\gamma}{ }^{-1}$. Hence the ring element $\gamma_{v} E_{\sigma \gamma}{ }^{-1}$ contains $g_{k}$ and $g_{k}^{-1}$ with the same coefficient.

3. Standard tableaux and idempotents. In a Young diagram [ $\lambda]$ having $\lambda_{i}$ nodes in row $i$ and $\lambda_{j}^{\prime}$ nodes in column $j$, we call $j-i$ the height of the $i j$ node, and we define the $i j$-hook $h_{i j}$ by the formula [3]

$$
h_{i j}=\left(\lambda_{i}-j\right)+\left(\lambda_{j}^{\prime}-i\right)+1 .
$$

In a two part Young diagram we shall add a large $w$ to each height in the second part, and replace $\lambda$ by $\mu$ in hook lengths of the second part. The products of the hook numbers for the nodes in $[\lambda],[\mu]$, and $[\lambda ; \mu]$ are the hook products $h^{\lambda}, h^{\mu}$, and $h^{\lambda \mu}=h^{\lambda} h^{\mu}$, respectively.

For example, for the partition $[\lambda ; \mu]=\left[3,2 ; 1^{2}\right]$ we have 


\begin{tabular}{|c|c|c|c|}
\hline Diagram $\left[3,2 ; 1^{2}\right]$ & Height graph & Hook graph & Hook products \\
\hline$[\lambda] \cdot \cdots \cdot$ & $\begin{array}{rrr}012 \\
-10\end{array}$ & $\begin{array}{lll}4 & 3 & 1 \\
2 & 1\end{array}$ & $h^{\lambda}=24$ \\
\hline$[\mu]$ & $\begin{array}{c}w \\
w-1\end{array}$ & $\begin{array}{l}2 \\
1\end{array}$ & $h^{\mu}=2, h^{\lambda \mu}=48$ \\
\hline
\end{tabular}

The associated diagram $\left[\lambda^{\prime} ; \mu^{\prime}\right]=\left[2^{2} 1 ; 2\right]$, obtained by interchanging rows and columns, has the same hook products, but has heights of opposite signs.

A standard Young tableau $t_{v}^{\lambda \mu}$ is obtained from a Young diagram by assigning the digits $d=1,2, \ldots, n$ to its nodes in any order that increases from left to right in rows and from top to bottom in columns. Since the number $f^{\lambda}$ of one part standard tableaux $t_{v}^{\lambda}$ is $f^{\lambda}=l ! / h^{\lambda}$, the number of two part standard tableaux $t_{v}^{\lambda \mu}=t_{v}^{\lambda} \oplus t_{v}^{\mu}$ is

$$
f^{\lambda \mu}=\left(\begin{array}{c}
n \\
m
\end{array}\right) f^{\lambda} f^{\mu}=n ! / h^{\lambda} h^{\mu}=n ! / h^{\lambda \mu} .
$$

Hence the required factor $h={ }^{o} G / f$ in $(2.8)$ is the hook product $h=h^{\lambda \mu}$.

The positive symmetric group $P_{v}^{\lambda \mu}$ associated with the standard tableau $t_{v}^{\lambda \mu}$ is the sum of all permutations on $n$ digits that leave each digit in the same row of the diagram as before. The negative symmetric group $N_{v}^{\lambda \mu}$ associated with $t_{v}^{\lambda \mu}$ is the sum of the even permutations minus the sum of the odd permutations that leave each digit in the same column as before. The product $P_{v}^{\lambda \mu} N_{v}^{\lambda \mu}$ is a weighted double coset that is a sum of permutations $g$ of $S_{n}$ with coefficients $\delta_{v}^{\lambda \mu}(g)$ that are $1,-1$, or 0 .

$$
P_{v}^{\lambda u} N_{v}^{\lambda u}=\sum_{g} \delta_{v}^{\lambda \mu}(g) g
$$

This ring element, used in generating Young's semi-normal representation (when $\mu=0$ ), is known [4] to be a multiple of an idempotent, and we have the relation

$$
\left(P_{v}^{\lambda \mu} N_{v}^{\lambda \mu}\right)^{2}=\left(P_{v}^{\lambda \mu} N_{v}^{\lambda \mu}\right) h^{\lambda \mu}
$$

Also, if the group sum $P_{1}^{\lambda \mu}$ is divided by its order ${ }^{o} P_{1}^{\lambda \mu}$, then $P_{1}^{\lambda \mu} /{ }^{o} P_{1}^{\lambda \mu}$ is an idempotent. We define $E^{\lambda \mu}$ to be the ring element

$$
E^{\lambda \mu}=P_{1}^{\lambda \mu} N_{1}^{\lambda \mu} P_{1}^{\lambda \mu} /{ }^{o} P_{1}^{\lambda \mu}
$$

in which inverse elements appear with the same coefficient. We see by (3.5) 
and (3.6) that $E^{\lambda \mu}$ satisfies the condition (2.8), since

$$
\left(E^{\lambda \mu}\right)^{2}=\left[P_{1}^{\lambda \mu} N_{1}^{\lambda \mu} P_{1}^{\lambda \mu} N_{1}^{\lambda \mu}\right] P_{1}^{\lambda \mu} /{ }^{o} P_{1}^{\lambda \mu}=E^{\lambda \mu} h^{\lambda \mu} .
$$

Next we construct the idempotent $\sigma^{\lambda \mu}$ from the normal subgroup $\sum_{n}$ of $G_{n}$

$$
\sigma^{\lambda \mu}=2^{-n} \prod_{d=1}^{l}\left(I+\sigma_{d}\right) \cdot \prod_{d=l+1}^{n}\left(I-\sigma_{d}\right)
$$

and the related idempotents to be associated with the other tableaux $t_{v}^{\lambda_{i}}$

$$
\sigma_{v}^{\lambda \mu}=2^{-n} \prod_{d \in t_{v}^{\lambda}}\left(I+\sigma_{d}\right) \cdot \prod_{d \in t_{v}^{\mu}}\left(I-\sigma_{d}\right)
$$

The idempotent $\sigma^{\lambda \mu}$ commutes with $P_{1}^{\lambda \mu}, N_{1}^{\lambda \mu}$, and $E^{\lambda \mu}$. Choosing $\gamma_{1}=I$, we take the product $E^{\lambda \mu} \sigma^{\lambda \mu}$ to be the 1, 1-entry of the group matrix $\mathfrak{M}$ in (1.2).

4. The ideal basis. The ring elements $\gamma_{\nu} E^{\lambda \mu} \sigma^{\lambda \mu}$ from the first row of the group matrix (1.2) should be a left ideal basis in $\Gamma$ for our irreducible orthogonal representation $\{\lambda ; \mu\}$ of $G_{n}$. The matrix of the transposition $\tau_{d}=$ $(d, d+1)$ in this representation is to have the $v v$-entry $\rho_{v}\left(\tau_{d}\right)$. If the digits $d+1$ and $d$ are assigned to the same part $t_{v}^{\lambda}$ or $t_{v}^{\mu}$ of the standard tableau $t_{v}^{\lambda \mu}$, then the integer $1 / \rho_{v}\left(\tau_{d}\right)$ is equal to the height of the digit $d+1$ minus the height of the digit $d$. If $d+1$ and $d$ are in different parts of $t_{v}^{\lambda \mu}$, we let $w$ in (3.2) become infinite, and define the reciprocal of the height difference to be $\rho_{\nu}\left(\tau_{d}\right)$ $=0$.

The $f^{\lambda \mu}$ standard tableaux are assigned a dictionary order, such that if the digits are read from left to right by rows, starting at the top row, the first digit in which two tableaux disagree will be larger for the later tableau. If $t_{u}^{\lambda \mu}$ precedes $t_{v}^{\lambda \mu}$ and the two tableaux differ only by the transposition of digits $d$ and $d+1$, we have $\rho_{v}\left(\tau_{d}\right)=-\rho_{u}\left(\tau_{d}\right)>0$, and we take

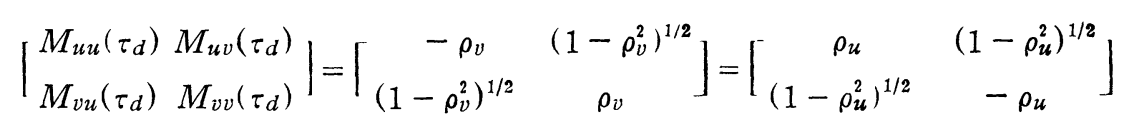

as in Young's orthogonal representation [4]. All other off-diagonal entries of $M^{\lambda \mu}\left(\tau_{d}\right)$ are set equal to 0 . The resulting real symmetric orthogonal matrix is a direct sum of $1 \times 1$ orthogonal matrices +1 or -1 , and $2 \times 2$ orthogonal matrices (4.1). On substituting the matrices (4.1), the condition (2.6) becomes 


$$
\begin{aligned}
& \tau_{d} \gamma_{u}=\rho_{u} \gamma_{u}+\left(1-\rho_{u}^{2}\right)^{1 / 2} \gamma_{v} \\
& \tau_{d} \gamma_{v}=\left(1-\rho_{v}^{2}\right)^{1 / 2} \gamma_{u}+\rho_{v} \gamma_{v}
\end{aligned} \quad \rho_{v}=-\rho_{u}>0 .
$$

These equations yield the following important formula

$$
\gamma_{v} \gamma_{u}^{-1}=\left(\tau_{d}-\rho_{u} I\right)\left(1-\rho_{u}^{2}\right)^{-1 / 2}=\left(\tau_{d}+\rho_{\nu} I\right)\left(1-\rho_{v}^{2}\right)^{-1 / 2} .
$$

The ring factor $\gamma_{v}$ for the tableau $t_{v}^{\lambda \mu}$ is hereby expressed in terms of the ring factor $\gamma_{u}$ for an earlier tableau $t_{u}^{\lambda \mu}$ that differs from $t_{v}^{\lambda \mu}$ only by the interchange of digits $d$ and $d+1$. Since $\gamma_{1}=I$, the factor $\gamma_{v}$ can be read off from the tableau $t_{v}^{\lambda \mu}$, by transposing pairs of consecutive digits until $t_{v}^{\lambda \mu}$ is reduced by one or more steps to $t_{1}^{\lambda \mu}$. For example, if

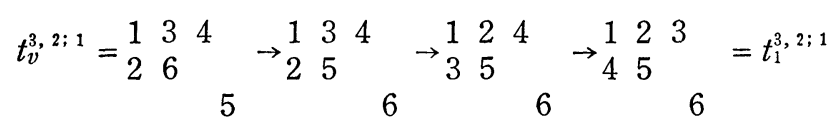

then

$$
\gamma_{v}=\frac{\tau_{5}+0 \cdot I}{1} \cdot \frac{\tau_{2}+I / 2}{(3 / 4)^{1 / 2}} \cdot \frac{\tau_{3}+I / 3}{(8 / 9)^{1 / 2}}
$$

The generators $\tau_{d}$ and $\sigma_{d}$ of $G_{n}$ satisfy the relations

$$
\sigma_{d} \tau_{d}=\tau_{d} \sigma_{d+1}, \text { and } \tau_{d}^{2}=\sigma_{d}^{2}=I
$$

It follows from (4.6) and (3.9) that

$$
\begin{aligned}
& \sigma_{v}^{\lambda \mu} \tau_{d}=\tau_{d} \sigma_{u}^{\lambda \mu}, \text { and } \sigma_{v}^{\lambda \mu}\left(\tau_{d}+\rho_{v} I\right)=\left(\tau_{d}+\rho_{v} I\right) \sigma_{u}^{\lambda \mu} \\
& \gamma_{v}^{-1} \sigma_{v}^{\lambda \mu} \gamma_{v}=\gamma_{u}^{-1} \sigma_{u}^{\lambda \mu} \gamma_{u}=\sigma^{\lambda \mu} .
\end{aligned}
$$

Thus condition (2.4) is satisfied, since

$$
\sigma_{d} \gamma_{v} E^{\lambda \mu} \sigma^{\lambda \mu}=\sigma_{d} \sigma_{v}^{\lambda \mu} \gamma_{v} E^{\lambda \mu}= \pm \gamma_{v} E^{\lambda \mu} \sigma^{\lambda \mu}
$$

where the sign is +1 or -1 according as digit $d$ belongs to $t_{v}^{\lambda}$ or $t_{v}^{\mu}$.

The construction described above verifies our main result.

Theorem. Every irreducible representation over the complex field of the hyperoctahedral group $G_{n}$ is similar to a real orthogonal representation $\{\lambda ; \mu\}$, whose group matrix $\mathfrak{M}^{\lambda \mu}$ has the $u v$-entry (1.2), where $E^{\lambda \mu}, \sigma^{\lambda \mu}$, and $\gamma_{v}$ are defined respectively by (3.6), (3.8), and (4.3).

For the symmetric group $S_{n}$ the corresponding irreducible orthogonal group matrices are obtained by taking $\mu=0$, and replacing $\sigma^{\lambda \mu}$ by $I$. 


\section{REFERENCES}

[1] Ferns, H. H., The Irreducible Representations of a Group and its Fundamental Regions, Trans. Roy. Soc. Can., Ser 3, Sec. III, vol. 28, 1934.

[2] Frame, J. S., The Constructive Reduction of Finite Group Representations, Proc. of Symposia in Pure Math. (1960 Institute on Finite Groups) vol. VI, pp. 89-99.

[3] Frame, J. S., Robinson, G. de B., and Thrall, R. M., The hook graphs of the symmetric group, Can. Jour. Math., vol. 6 (1954), pp. 316-324.

[4] Robinson, G. de B., Representation Theory of the Symmetric Group, University of Toronto Press, 1961. Especially pp. 35-38.

Michigan State University 